Pacific Journal of Mathematic 


\title{
EQUATIONS OF MEAN CURVATURE TYPE IN 2 INDEPENDENT VARIABLES
}

\author{
LEON SIMON
}

The object of this paper is to develop a regularity theory for equations of mean curvature type in two independent variables. An equation of mean curvature type in two independent variables is defined to be an equation of the form

$$
\sum_{i, j=1}^{2} a_{i j}(x, u, D u) D_{i j} u=b(x, u, D u)
$$

on a domain $\Omega \subset R^{2}$, where the functions $a_{i j}, b$ satisfy special structural conditions. Namely, we require that (i) $\left(1+|D u|^{2}\right)^{-1 / 2} b(x, u, D u)$ is bounded by a fixed constant (independent of $u$ ), and (ii) the quadratic form $\sum_{i, j=1}^{2} a_{i j}(x, u, D u) \xi_{i} \xi_{j}$ is bounded from above and below in terms of the quadratic form $\sum_{i, j=1}^{2} g^{i j}(D u) \xi_{i} \xi_{j}$, where $g^{i j}(D u)=\delta_{i j}-D_{i} u D_{j} u /\left(1+|D u|^{2}\right)$, $i, j=1,2$, are the coefficients of the minimal surface equation.

R. Finn [2] was the first to consider such equations; he considered the case $b \equiv 0$ and $a_{i j}(x, u, D u) \equiv a_{i j}(D u)$. Later Jenkins [5] and Jenkins-Serrin [6] specialized further to equations which arise as the non-parametric Euler-Lagrange equation of a parametric elliptic functional with integrand independent of the spatial variables (see Appendix 1). The main results in [2] concerned a-priori estimates for the gradient of a solution. In [5], [6] somewhat deeper results were obtained; in particular, pointwise estimates for the principal curvatures of the graph of a solution were established. Recently J. Spruck [11] obtained such a pointwise curvature estimate for the constant mean curvature equation; this was the first such result obtained for a non-homogeneous (i.e. $b$ not identically zero) equation of mean curvature tpye.

In this paper we intend to use the Hölder estimate established in [8] in order to obtain a strong regularity theory for the entire class of equations of mean curvature type. The plan of the paper is as follows. In $\S 1$ we introduce the class of equations of mean curvature type and give a geometric characterization of such equations. In $\S 2$ we discuss application of the results of [8] to homogeneous equations of mean curvature type; in particular we obtain some a-priori gradient estimates, a Bernstein type theorem, a Bers-type theorem concerning the limiting behaviour of the gradient of solutions defined outside a compact set, a global Hölder continuity estimate for solutions which continuously attain Lipschitz boundary 
values on $\partial \Omega$, a pointwise estimate for the principal curvatures of the graph of a solution, and a theorem concerning the removability of isolated singularities. Except for the pointwise curvature estimate, all of these results are obtained without any continuity restrictions on the coefficient functions $a_{i j}$. In $\S 3$ we discuss extensions of the results of $\S 2$ to the nonhomogeneous case.

1. Preliminaries. By an equation of mean curvature type, we mean an equation of the form

$$
\sum_{i, j=1}^{2} a_{i j}(x, u, D u) D_{i j} u=b(x, u, D u)
$$

on a domain $\Omega \subset \boldsymbol{R}^{2}$, where $a_{i j}, i, j=1,2$, and $b$ are given real-valued functions on $\Omega \times \boldsymbol{R} \times \boldsymbol{R}^{2}$ with

$$
|\xi|^{2}-\frac{(\xi \cdot p)^{2}}{1+|p|^{2}} \leqq \sum_{i, j=1}^{2} a_{i j}(x, z, p) \xi_{2} \xi_{j} \leqq \gamma\left(|\xi|^{2}-\frac{(\xi \cdot p)^{2}}{1+|p|^{2}}\right)
$$

for all $(x, z, p) \in \Omega \times R \times R^{2}$ and all $\xi=\left(\xi_{1}, \xi_{2}\right) \in R^{2}$; and

$$
|b(x, z, p)| \leqq \mu \sqrt{1+|p|^{2}}
$$

for all $(x, z, p) \in \Omega \times \boldsymbol{R} \times \boldsymbol{R}^{2}$. Here $\gamma$ and $\mu$ denote fixed constants. Note that the minimal surface equation can be written in the form (1.1) with $\alpha_{i j}(x, z, p)=\delta_{i j}-p_{i} p_{j} /\left(1+|p|^{2}\right)$ and with $b \equiv 0$. In this case (1.2), (1.3) hold with $\gamma=1$ and $\mu=0$. More generally, any equation which arises as the non-parametric Euler-Lagrange equation of a parametric elliptic functional (see Appendix 1) is of the form (1.1), (1.2), (1.3). But quite apart from these examples, the equations of mean curvature type are both natural and interesting in that they are completely characterized as follows:

Suppose $u$ is a $C^{2}(\Omega)$ function with graph

$$
M=\{(x, z): x \in \Omega, z=u(x)\} .
$$

Then there exists real-valued functions $a_{i j}, b$ such that (1.1)-(1.3) hold if and only if the principal curvatures $\kappa_{1}, \kappa_{2}$ of $M$ are related at each point of $M$ by an equation of the form

$$
\alpha_{1} \kappa_{1}+\alpha_{2} \kappa_{2}=\beta,
$$

with $\alpha_{1}, \alpha_{2}, \beta$ satisfying$$
1 \leqq \alpha_{i} \leqq \gamma, \quad i=1,2,
$$

$$
|\beta| \leqq \mu .
$$

To demonstrate that this characterization is valid, we let $d$ denote 
the distance function of $M$ defined for $X=(x, z) \in \Omega \times R$ by setting $d(X)=\operatorname{dist}(X, M)$ if $z>u(x)$ and $d(X)=-\operatorname{dist}(X, M)$ if $z<u(x)$. Since $d$ is $C^{2}$ and $d(x, u(x)) \equiv 0, x \in \Omega$, we then have, by the chain rule, the identities $D_{i} d(X)+D_{i} u(x) D_{3} d(X)=0$ and

$$
\begin{aligned}
D_{i j} d(X) & +D_{i} u(x) D_{3 j} d(X)+D_{j} u(x) D_{3 i} d(X) \\
& +D_{i} u(x) D_{j} u(x) D_{33} d(X)+D_{3} d(X) D_{i j} u(x)=0,
\end{aligned}
$$

$i, j=1,2$, where $X=(x, u(x))$. Since $D_{3} d(X)=v^{-1}, v=\sqrt{1+|D u(x)|^{2}}$, (1.1) then implies

$$
\sum_{i, j=1}^{3} \alpha_{\imath j}^{*}(x) D_{i j} d(X)+b^{*}(x)=0,
$$

where $b^{*}(x)=v^{-1} b(x, u(x), D u(x))$ and where the $3 \times 3$ matrix $\left(a_{i j}^{*}(x)\right)$ is defined by setting $a_{i j}^{*}(x)=a_{i j}(x, u(x), D u(x))$ for $i, j=1,2$ and

$$
\begin{aligned}
a_{i 3}^{*}(x) & =a_{3 i}^{*}(x)=\sum_{j=1}^{2} D_{j} u(x) a_{i j}^{*}(x), \quad i=1,2, \\
a_{33}^{*} & =\sum_{i, j=1}^{2} D_{i} u(x) D_{j} u(x) a_{i j}^{*}(x) .
\end{aligned}
$$

Note that these last relations are equivalent to

$$
\sum_{j=1}^{3} a_{j i}^{*}(x) \nu_{j}=\sum_{j=1}^{3} a_{i j}^{*}(x) \nu_{j}=0, \quad i=1,2,3,
$$

where $\nu=v^{-1}(-D u(x), 1)(=D d(X))$ is the upward unit normal of $M$. Next we let $Q$ be the matrix with rows $e_{1}, e_{2}, \nu$, where $e_{1}, e_{2}$ are principal directions of $M$ at $X$, so that $Q\left(D_{i j} d(X)\right) Q^{t}=\operatorname{diag}\left[\kappa_{1}, \kappa_{2}, 0\right]$, where $\kappa_{1}, \kappa_{2}$ are principal curvatures of $M$ at $X$. Thus (1.5) can be written in the form (1.1)', with $\alpha_{1}, \alpha_{2}$ the first two elements on the leading diagonal of $Q\left(\alpha_{2 j}^{*}(x)\right) Q^{t}$ and with $\beta=b^{*}(x)$. (1.3)' is now true by (1.3). To check (1.2)', we first note that, by (1.6),

$$
\sum_{i, j=1}^{3} a_{i j}^{*}(x) \xi_{i} \xi_{j}=\sum_{i, j=1}^{2} a_{i j}^{*}(x)\left(\xi_{i}+\xi_{3} D_{i} u(x)\right)\left(\xi_{j}+\xi_{3} D_{i} u(x)\right), \quad \xi \in \boldsymbol{R}^{3},
$$

and it then follows from (1.2) that

$$
\left|\xi^{\prime}\right|^{2} \leqq \sum_{i, j=1}^{3} a_{i j}^{*}(x) \xi_{i} \xi_{j} \leqq \gamma\left|\xi^{\prime}\right|^{2}, \quad \xi^{\prime}=\xi-(\nu \cdot \xi) \nu,
$$

where $\nu=v^{-1}(-D u(x), 1)$. (1.2)' easily follows from this.

To prove the converse implication we suppose that $(1.1)^{\prime},(1.2)^{\prime}$, $(1.3)^{\prime}$ hold at $X=(x, u(x)) \in M$, we let $\left(a_{i j}^{*}(x)\right)=Q^{t} \operatorname{diag}\left[\alpha_{1}, \alpha_{2}, 0\right] Q$, where $Q$ is as above, and let $b^{*}(x)=\beta$. Then (1.5) holds and consequently, since we still have the relations (1.6), (1.6)', an application of (1.4) yields 


$$
\sum_{i, j=1}^{2} \alpha_{\imath j}^{*}(x) D_{i j} u+b^{*}(x)=0 \text {. }
$$

We then define, for $i, j=1,2$,

$$
a_{\imath j}(x, z, p)= \begin{cases}a_{\imath \jmath}^{*}(x) & \text { if } z=u(x) \text { and } p=D u(x) \\ \delta_{i j}-\frac{p_{i} p_{j}}{1+|p|^{2}} & \text { otherwise }\end{cases}
$$

and

$$
b(x, z, p)= \begin{cases}b^{*}(x) & \text { if } z=u(x) \text { and } p=D u(x) \\ 0 & \text { otherwise }\end{cases}
$$

(1.1) (1.3) are now easily checked.

Notice that if we square each side of (1.1)' and divide by $\alpha_{1} \alpha_{2}$, then we obtain

$$
\frac{\alpha_{1}}{\alpha_{2}} \kappa_{1}^{2}+\frac{\alpha_{2}}{\alpha_{1}} \kappa_{2}^{2}=-2 \kappa_{1} \kappa_{2}+\frac{\beta^{2}}{\alpha_{1} \alpha_{2}},
$$

and by $(1.2)^{\prime},(1.3)^{\prime}$ this gives

$$
\kappa_{1}^{2}+\kappa_{2}^{2} \leqq \Lambda_{1} \kappa_{1} \kappa_{2}+\Lambda_{2}, \quad \Lambda_{1}=-2 \gamma, A_{2}=\gamma \mu^{2} .
$$

This last inequality asserts precisely that the Gauss map of the graph of $M$ is $\left(\Lambda_{1}, A_{2}\right)$-quasiconformal in the sense of [8]. (See [8], (1.8), (1.9).) In particular the Gauss map is $\left(\Lambda_{1}, 0\right)$-quasiconformal, with $\Lambda_{1}=-2 \gamma$, in case $b \equiv 0$ (for then we can set $\mu=0$ ). These observations are the key in applying the results of [8] to the equations of mean curvature type.

2. The homogeneous case $(b \equiv 0)$. Throughout this section it is assumed that $u$ is a $C^{2}(\Omega)$ solution of (1.1), that $b \equiv 0$ on $\Omega \times R \times$ $R^{2}$, and that (1.2) holds.

$M$ will denote the graph of $u$; that is

$M=\{X=(x, u(x)): x \in \Omega\}$.

$v$ and $\nu=\left(\nu_{1}, \nu_{2}, \nu_{3}\right)$ will denote the functions defined on $M$ by $v(X)=\sqrt{1+|D u(x)|^{2}}, \quad X=(x, u(x)) \in M$

and

$$
\nu(X)=v^{-1}(-D u(x), 1), \quad X=(x, u(x)) \in M .
$$

(Thus $\nu$ is just the upward unit normal of $M$.) 
$\kappa_{1}(X), \kappa_{2}(X)$ will denote the principal curvatures of $M$ at $X$.

$x_{0}$ will denote a fixed point of $\Omega$.

$X_{0}$ will denote the point $\left(x_{0}, u\left(x_{0}\right)\right) \in M$.

$D_{\rho}\left(x_{0}\right)=\left\{x \in R^{2}:\left|x-x_{0}\right|<\rho\right\}$;

$S_{\rho}\left(X_{0}\right)=\left\{X \in M:\left|X-X_{0}\right|<\rho\right\}=M \cap B_{\rho}\left(X_{0}\right)$,

where

$B_{\rho}\left(X_{0}\right)=\left\{X \in R^{3}:\left|X-X_{0}\right|<\rho\right\}$.

We will begin by listing some results which follow directly from [8] $\S 3,4$ (by virtue of the remarks at the end of $\S 1$ above).

THEOREM 1. (See Theorem (4.2) of [8].) If $D_{\rho}\left(x_{0}\right) \subset \Omega$, then

$$
\sup _{S_{\rho / 2}\left(X_{0}\right)} v \leqq \underset{S_{\rho / 2}\left(X_{0}\right)}{c \inf } v
$$

where $c>0$ depends only on $\gamma$.

Theorem 2. (See Corollary (4.2) of [8].) If $u \geqq 0$ on $D_{\rho}\left(x_{0}\right)$, then

$$
\left|D u\left(x_{0}\right)\right| \leqq c_{1} \exp \left\{c_{2} u\left(x_{0}\right) / \rho\right\} ，
$$

whepe $c_{1}, c_{2}$ depend only on $\gamma$.

THEOREM 3. (See Theorem (4.3) of [8]). If $D_{\rho}\left(x_{0}\right) \subset \Omega$, then

$$
|\nu(X)-\nu(\bar{X})| \leqq c\left(v\left(X_{0}\right)\right)^{-1}\left\{\frac{|X-\bar{X}|}{\rho}\right\}^{\alpha}, \quad X, \bar{X} \in S_{\rho / 2}\left(X_{0}\right),
$$

where $c>0$ and $\alpha \in(0,1)$ depend only on $\gamma$.

THEOREM 4. (See Theorem (4.1) of [8]). If $\Omega=R^{2}$, then $u$ is linear.

(Note that Theorem 4 follows directly from Theorem 3 by letting $\rho \rightarrow \infty$.)

TheOREM 5. (See Theorem (4.4) of [8].) Suppose $u$ extends continuously to $\bar{\Omega}$, suppose $\varphi$ is a Lipschitz function on $\boldsymbol{R}^{2}$ with $\sup |D \varphi| \leqq L$. Then, if $\lim _{\substack{x \rightarrow y \\ x \in d}} u(x)=\varphi(y)$ at each $y \in \partial \Omega$, we have

$$
|u(x)-u(\bar{x})| \leqq c\left\{M^{1-\alpha}+|x-\bar{x}|^{1-\alpha}\right\}|x-\bar{x}|^{\alpha}, \quad x, \bar{x} \in \Omega
$$

where $M=\sup _{\Omega}|u-\varphi|$ and $c>0, \alpha \in(0,1)$ are constants depending only on $L$. 
Notice that there is absolutely no dependence on the domain $\Omega$ in the estimate above. We should point out also that from Theorem 5 various other continuity estimates follow. (See Theorems 3 and 4 of [10].)

We now wish to mention some additional results which do not quite directly follow from [8]. First we have the following theorem, which is an analogue of a theorem established by Bers [1] for solutions of the minimal surface equation.

TheOREM 6. Suppose $\Omega=\boldsymbol{R}^{2} \sim K$, where $K$ is compact. Then there is a vector $a \in \boldsymbol{R}^{2}$ such that $D u(x) \rightarrow a$ uniformly for $|x| \rightarrow \infty$.

A somewhat stronger result than Theorem 6 will be established in Theorem $6^{\prime}$ of $\S 2$; in Theorem $6^{\prime}$ the condition that $b \equiv 0$ will be replaced by the requirement that $b$ has sufficiently rapid convergence to zero as $|x| \rightarrow \infty$.

Next we have a theorem concerning removability of isolated singularities. Such a theorem was proved by Bers [1] for solutions of the minimal surface equations and by Finn [3] for a class of divergence-form equations.

THEOREM 7. Suppose $D_{\rho}\left(x_{0}\right) \sim\left\{x_{0}\right\} \subset \Omega$. Then $u$ extends to be $a$ $C^{1, \alpha}\left(D_{\rho}\left(x_{0}\right)\right) \cap W^{2,2}\left(D_{\rho}\left(x_{0}\right)\right)$ function, where $\alpha \in(0,1)$ depends only on $\gamma$.

For a proof of this theorem the reader is referred to [9].

We will conclude this section with a pointwise curvature estimate of a type that was established by Heinz [4] for solutions of the minimal surface equation and by Jenkins [5] and Jenkins-Serrin [6] for a special class of equations of mean curvature type. In order to conveniently describe the restrictions on the coefficient functions $a_{i j}$ which are needed here, it is necessary to introduce some further notation. We define a $3 \times 3$ matrix $\left(a_{\imath j}^{*}(X, \mu)\right)$, where $X=(x, z) \in$ $\Omega \times R$ and $\mu=\left(1+|p|^{2}\right)^{-1 / 2}(-p, 1)$ with $p \in \boldsymbol{R}^{2}$, by

$$
\left\{\begin{array}{l}
a_{i j}^{*}(X, \mu)=a_{i j}(x, z, p), \quad i, j=1,2, \\
a_{3 i}^{*}(X, \mu)=a_{i 3}^{*}(X, \mu)=\sum_{j=1}^{2} a_{i j}(x, z, p) p_{j}, \\
a_{33}^{*}(X, \mu)=\sum_{i, j=1}^{2} a_{i j}(x, z, p) p_{i} p_{j} .
\end{array}\right.
$$

(Cf. the functions $a_{i j}^{*}(x)$ of $(1.6),(1.6)^{\prime}$.) Notice that $a_{i j}^{*}(X, \mu)$ is thus defined for $\mu \in S_{+}^{2}$, where

$$
S_{+}^{2}=\left\{q=\left(q_{1}, q_{2}, q_{3}\right) \in \boldsymbol{R}^{3}:|q|=1, q_{3}>0\right\} .
$$


In the case when (1.1) arises as the nonparametric Euler-Lagrange equation of a parametric elliptic elliptic functional, the matrix $\left(a_{2 j}^{*}(X, \mu)\right)$ arises quite naturally (see Appendix 1).

TheOREM 8. Suppose $D_{\rho}\left(x_{0}\right) \subset \Omega$ and

$$
\sum_{i, j=1}^{3}\left|a_{i j}^{*}(X, \mu)-a_{i j}^{*}(\bar{X}, \bar{\mu})\right| \leqq \delta\{|X-\bar{X}| / \rho+|\mu-\bar{\mu}|\}^{\alpha}
$$

for all $X, \bar{X} \in S_{\rho}\left(X_{0}\right)$ and all $\mu, \bar{\mu} \in S_{+}^{2}$, where $\delta>0$ and $\alpha \in(0,1)$ are constants. Then

$$
\left(\kappa_{1}^{2}+\kappa_{2}^{2}\right)\left(X_{0}\right) \leqq c\left(v\left(X_{0}\right)\right)^{-2} \rho^{-2},
$$

where $c$ is a constant depending only on $\gamma, \alpha$ and $\delta$.

Proof. For sufficiently small $\theta \in(0,1)$, depending on $\gamma$, we know $S_{\theta \rho}\left(X_{0}\right)$ is connected by [8], Lemma $(3.2)^{1}$. Let $(\xi, \zeta)=\left(\xi_{1}, \xi_{2}, \zeta\right)$ denote new coordinates for $\boldsymbol{R}^{3}$ defined by

$$
(\xi, \zeta)=\left(X-X_{0}\right) Q^{t},
$$

where $Q$ is an orthogonal matrix with rows $e_{1}, e_{2}, \nu\left(X_{0}\right)$, with $\left\{e_{1}, e_{2}\right\}$ any orthonormal basis for the tangent space of $M$ at $X_{0}$. By the Hölder estimate of Theorem 3 it is clear that there is a $\theta \in(0,1)$, depending only on $\gamma$, such that $S_{\theta \rho}\left(X_{0}\right)$ can be represented, relative to the new coordinates $(\xi, \zeta)$, in the form

$$
\zeta=\widetilde{u}(\xi), \quad \xi \in U,
$$

where $U$ is an open subset of $R^{2}, \tilde{u} \in C^{2}(U)$ and

$$
\sup |D \tilde{u}| \leqq 1, \quad D_{\theta \rho}(0) \subset U, \quad \widetilde{u}(0)=0 .
$$

Furthermore, again using Theorem 3 we can infer that

$$
|D \widetilde{u}(\xi)-D \widetilde{u}(\bar{\xi})| \leqq c\left(v\left(X_{0}\right)\right)^{-1}\{|\xi-\bar{\xi}| / \rho\}^{\alpha}, \quad \xi, \bar{\xi} \in D_{\theta \rho}(0),
$$

provided $\theta \in(0,1)$ is sufficiently small (depending on $\gamma$ ). By the discussion of $\S 1$ we can also infer from (1.1) and (1.2) that $\widetilde{u}$ satisfies an equation of the form

$$
\sum_{i, j=1}^{2} \widetilde{a}_{i j}(\xi) D_{i j} \widetilde{u}=0 \text { on } U
$$

where

1 We will henceforth use this connectivity result whenever it is convenient to do so; note that for the inhomogeneous case the choice of $\theta$ depends also on $\mu \rho$. 


$$
|\lambda|^{2}-\frac{(\lambda \cdot D \tilde{u})^{2}}{1+|D \tilde{u}|^{2}} \leqq \widetilde{a}_{i j}(\xi) \lambda_{i} \lambda_{j} \leqq \gamma\left(|\lambda|^{2}-\frac{(\lambda \cdot D \tilde{u})^{2}}{1+|D \tilde{u}|^{2}}\right)
$$

for all $\lambda \in R^{2}$ and $\xi \in U$. By (2.6) this clearly implies

$$
\frac{1}{2}|\lambda|^{2} \leqq \sum_{i, j=1}^{2} \widetilde{a}_{i j}(\xi) \lambda_{i} \lambda_{j} \leqq \gamma|\lambda|^{2}
$$

(because $|\lambda|^{2}-(\lambda \cdot D \tilde{u})^{2} /\left(1+|D \tilde{u}|^{2}\right) \geqq|\lambda|^{2} /(1+|D \tilde{u}|)^{2}$ by Cauchy's inequality). In fact by virtue of the discussion of $\S 1$ together with (2.7) and the the Hölder condition (2.3), it is clear that we may assume

$$
\left|\tilde{a}_{i j}(\xi)-\tilde{a}_{i j}(\bar{\xi})\right| \leqq c\{|\xi-\bar{\xi}| / \rho\}^{\tau}, \quad \xi, \bar{\xi} \in D_{\theta \rho}(0),
$$

where $c>0$ and $\tau \in(0,1)$ depend only on $\alpha, \delta$ and $\gamma$.

Now by (2.9), (2.10) and the Schauder interior estimate for solutions of (2.8), we then have

$$
\left\{\sum_{i, j=1}^{2}\left(D_{i j} \widetilde{u}(0)\right)^{2}\right\}^{1 / 2} \leqq c \rho^{-2} \sup _{D_{\theta \rho}(0)}|\widetilde{u}|,
$$

where $c$ depends only on $\gamma, \alpha$ and $\delta$. On the other hand, since $D \tilde{u}(0)=0$ and $\tilde{u}(0)=0$, we deduce from (2.7) that

$$
\sup _{D_{\theta \rho}(0)}|\tilde{u}| \leqq c \rho\left(v\left(X_{0}\right)\right)^{-1},
$$

where $c$ depends only on $\gamma$. Also, again using the fact that $D \widetilde{u}(0)=0$, we have

$$
\sum_{i, j=1}^{2}\left(D_{i j} \tilde{u}(0)\right)^{2}=\left(\kappa_{1}^{2}+\kappa_{2}^{2}\right)\left(X_{0}\right) .
$$

The theorem is now proved by combining (2.11), (2.12) and (2.13).

3. The inhomogeneous case. Here the notation will be the same as in $\S 2$, except that (1.3) is assumed in place of the condition $b \equiv 0$.

All the results of $\S 2$, except Theorem 4 , have analogues in this more general setting, but in most cases either the hypotheses on the coefficient functions $a_{i j}$ must be stronger or the conclusion weaker than for the corresponding results of $\S 2$.

We first have the following analogue of Theorem 3 .

TheOREM 3'. If $D_{\rho}\left(x_{0}\right) \subset \Omega$, then

$$
|\nu(X)-\nu(\bar{X})| \leqq c\left\{\frac{|X-\bar{X}|}{\rho}\right\}^{\alpha}, \quad X, \bar{X} \in S_{\rho / 2}\left(X_{0}\right),
$$

where $c>0$ and $\alpha \in(0,1)$ depend only on $\gamma$ and $\mu \rho$. 
In view of the remarks at the end of $\S 1$, this theorem is a special case of [8], Theorem (3.1). By considering examples it is easy to see that an estimate like that of Theorem 3, with the factor $\left(v\left(X_{0}\right)\right)^{-1}$ on the right hand side, cannot hold in the present inhomogeneous setting. (One class of examples is obtained by considering the constant mean curvature equation

$$
\sum_{i=1}^{2} D_{i} \frac{D_{i} u}{\sqrt{1+|D u|^{2}}}=H
$$

where $H$ is any nonzero constant.)

For the purposes of the present section it will be convenient to define the function $b^{*}(X, \mu)$ for

$$
X=(x, z) \in \Omega \times R \text { and } \mu=\frac{(-p, 1)}{\sqrt{1+|p|^{2}}}, \quad p \in R^{2},
$$

by

$$
b^{*}(X, \mu)=\left(1+|p|^{2}\right)^{-1 / 2} b(x, z, p) .
$$

Note that $b^{*}$ is thus well defined for $\mu \in S_{+}^{2}$, with $S_{+}^{2}$ as in (2.2). (The function $b^{*}$, like the functions $a_{i j}^{*}$ of (2.1), arise quite naturally in case (1.1) is the non-parametric Euler-Lagrange equation of a parametric elliptic functional-see Appendix 1.)

Next we want to obtain analogues of Theorems 1,2 for the inhomogeneous case. We will impose the following restrictions on the functions $a_{i j}^{*}, b^{*}$ :

$$
\begin{aligned}
& \rho \sqrt{1+|p|^{2}}\left|\frac{\partial a_{i j}^{*}}{\partial z}(x, z, \mu)\right|+\rho \sum_{k=1}^{2}\left|\frac{\partial}{\partial x_{k}} a_{i j}^{*}(x, z, \mu)\right| \\
& +|\mu-\bar{\mu}|^{-1}\left|a_{i j}^{*}(x, z, \mu)-a_{i j}^{*}(x, z, \bar{\mu})\right| \leqq \delta \\
& \rho \sqrt{1+|p|^{2}}\left|\frac{\partial b^{*}}{\partial z}(x, z, \mu)\right|+\rho \sum_{k=1}^{2}\left|\frac{\partial}{\partial x_{k}} b^{*}(x, z, \mu)\right| \\
& +|\mu-\bar{\mu}|^{-1}\left|b^{*}(x, z, \mu)-b^{*}(x, z, \bar{\mu})\right| \leqq \delta \rho^{-1}
\end{aligned}
$$

for all $(x, z) \in \Omega \times R$ and $\mu, \bar{\mu} \in S_{+}^{2}$ with $\mu \neq \bar{\mu}$ and

$$
\mu=\frac{(-p, 1)}{\sqrt{1+|p|^{2}}}
$$

In these inequalities $\delta$ denotes a fixed constant.

THEOREM 1'. If $D_{\rho}\left(x_{0}\right) \subset \Omega$ and if (3.2), (3.3) hold, then

$$
\sup _{S_{\rho / 2}\left(X_{0}\right)} v \leqq c \inf _{S_{\rho / 2}\left(X_{0}\right)} v
$$


where $c>0$ depends only on $\gamma, \mu \rho, \delta$.

Before giving the proof of this theorem we point out that, by an argument like that used to prove Theorem 2, we can infer the following from Theorem 1'.

THEOREM 2'. Under the hypotheses of Theorem 1',

$$
\left|D u\left(x_{0}\right)\right| \leqq c_{1} \exp \left\{c_{2} u\left(x_{0}\right) / \rho\right\} ，
$$

provided $u \geqq 0$ on $D_{\rho}\left(x_{0}\right)$. Here $c_{1}, c_{2}$ are constants depending on $\gamma, \mu \rho$ and $\delta$.

Proof of Theorem 1'. We consider two cases:

Case I. $\left|D u\left(x_{0}\right)\right| \leqq 2$. In this case the Hölder estimate of Theorem $3^{\prime}$ can be used to deduce $\sup _{D_{\rho \theta}\left(x_{0}\right)}|D u| \leqq 3$ for suitable $\theta \in(0,1)$ depending only on $\gamma$ and $\mu \rho$. Hence the required result is established in this case.

Case II. $D u\left(x_{0}\right)>2$. In this case we introduce new coordinates $(\xi, \zeta)$ as in (2.4), where now $Q$ has the form

$$
Q=\left(\begin{array}{lcl}
0 & 0 & 1 \\
\cos \alpha & -\sin \alpha & 0 \\
\sin \alpha & \cos \alpha & 0
\end{array}\right)
$$

for some constant $\alpha$ to be chosen. Since $\left|D u\left(x_{0}\right)\right|>2$, which guarantees $\nu\left(X_{0}\right) \cdot(0,0,1)<1 / \sqrt{5}$, it is clear from the Hölder estimate of Theorem $3^{\prime}$ that $\alpha$ can be chosen such that there is a representation of the form (2.5), (2.6) for suitable $\theta \in(0,1)$. Also by (1.1)-(1.3) and by the discussion of $\S 1$, we know that

$$
\sum_{i, j=1}^{2} \tilde{a}_{i j}(\xi) D_{i j} \tilde{u}=\tilde{b}(\xi) \text { on } U
$$

(cf. (2.8)), where (2.9) holds and where $|\widetilde{b}(\xi)| \leqq \mu$ on $U$. Here $U$ is as in (2.5).

Now let $\zeta=\zeta(\xi)$ be a $C^{1}$ function with compact support in $U$, multiply by $D_{1} \zeta$ in (3.5) and integrate over $U$. After making use of the relations

$$
\int_{U} D_{22} \tilde{u} D_{1} \zeta d \xi=-\int_{U} D_{2} \tilde{u} D_{12} \zeta d \xi=\int_{U} D_{21} \tilde{u} D_{2} \zeta,
$$


this gives

$$
\int_{U i, j=1}^{2} \alpha_{i j} D_{i} \psi D_{j} \zeta d \xi=\int_{U}\left(D_{1} \beta\right) \zeta d \xi
$$

where $\psi=D_{1} \tilde{u}, \alpha_{11}=\widetilde{a}_{11} / \widetilde{a}_{22}, \alpha_{22} \equiv 1, \alpha_{12} \equiv 0, \alpha_{21}=2 \widetilde{a}_{12} / \widetilde{a}_{22}$, and $\beta=\tilde{b} / \widetilde{a}_{22}$. Thus $\psi=D_{1} \tilde{u}$ is a weak solution of the equation

$$
\sum_{i, j=1}^{2} D_{i}\left(\alpha_{i j} D_{j} \psi\right)=-D_{1} \beta
$$

Since (2.4) and (3.4) imply the relations

$$
\begin{aligned}
\xi_{1} & =u(x)-u\left(x_{0}\right), \\
x-x_{0} & =\left(\xi_{1} \cos \alpha+\tilde{u}(\xi) \sin \alpha,-\xi_{2} \sin \alpha+\tilde{u}(\xi) \cos \alpha\right),
\end{aligned}
$$

one can easily check that

$$
\begin{aligned}
D_{1} \beta= & -\widetilde{a}_{22}^{-2}(\xi) b^{*}(x, u, \nu) \sum_{i, j=1}^{3} \lambda_{i} \lambda_{j}\left\{\frac{\partial}{\partial z} a_{i j}^{*}(x, u, \nu)\right. \\
& +\frac{\partial}{\partial x_{1}} a_{i j}^{*}(x, u, \nu) \sin \alpha D_{1} \tilde{u}(\xi)+\frac{\partial}{\partial x_{2}} a_{i j}^{*}(x, u, \nu) \cos \alpha D_{1} \tilde{u}(\xi) \\
& \left.+\frac{\partial}{\partial \mu} a_{i j}^{*}(x, u, \nu) \cdot D_{1} \tilde{\nu} Q\right\}+\tilde{a}_{22}^{-1}(\xi)\left\{\frac{\partial}{\partial z} b^{*}(x, u, \nu)\right. \\
& +\frac{\partial}{\partial x_{1}} b^{*}(x, u, \nu) \sin \alpha D_{1} \tilde{u}(\xi)+\frac{\partial}{\partial x_{2}} b^{*}(x, u, \nu) \cos \alpha D_{1} \tilde{u}(\xi) \\
& \left.+\frac{\partial}{\partial \mu} b^{*}(x, u, \nu) \cdot D_{1} \tilde{\nu} Q\right\} .
\end{aligned}
$$

Here $\lambda=\left(\lambda_{1}, \lambda_{2}, \lambda_{3}\right)$ denotes the second row of $Q$ (so that $\tilde{a}_{22}=$ $\sum_{i, j=1}^{3} a_{i j}^{*} \lambda_{i} \lambda_{j}$ in accordance with the discussion of $\S 1$ ). Also, $\tilde{\nu}=$ $(-D \tilde{u}, 1) / \sqrt{1+|D \tilde{u}|^{2}}, \partial / \partial \mu$ denotes the gradient operator on $S_{+}^{2}$, and we have used the relation $\widetilde{\nu} Q=\nu$. Then, since

$$
D_{1} \widetilde{\nu}_{k}=\left(1+|D \tilde{u}|^{2}\right)^{-1 / 2} \sum_{j=1}^{2}\left(\delta_{k j}-\widetilde{\nu}_{k} \widetilde{\nu}_{j}\right) D_{j_{1}} \tilde{u}
$$

we see (by using the conditions (3.2), (3.3) together with (2.6) and the identity $D_{1} \tilde{u} / \sqrt{1+|\bar{D} \tilde{u}|^{2}}=v^{-1}$ ) that (3.6) implies

$$
\int_{U}\left(\sum_{i, j=1}^{2} \alpha_{i j} D_{j} \psi D_{i} \zeta+\sum_{i=1}^{2} \beta_{i} D_{i} \psi \zeta+\tau \psi \zeta\right) d \xi=0, \quad \zeta \in C_{0}^{1}(U),
$$

where

$$
\begin{gathered}
\frac{1}{2}|\theta|^{2} \leqq \sum_{j, i=1}^{2} \alpha_{i j} \theta_{i} \theta_{j} \leqq \gamma|\theta|^{2}, \quad \theta=\left(\theta_{1}, \theta_{2}\right) \in \boldsymbol{R}^{2} ; \text { and } \\
\rho \sum_{i=1}^{2}\left|\beta_{i}\right|+\rho^{2}|\tau| \leqq c_{1}
\end{gathered}
$$


where $c_{1}$ depends only on $\mu \rho$ and $\delta$. Clearly we can apply the De Giorgi, Nash, Moser theory and deduce that

$$
\sup _{D_{\theta \rho / 2}(0)} \psi \leqq c_{2} \inf _{D_{\theta \rho / 2}(0)} \psi
$$

for non-negative solutions $\psi$ of (3.7), where $c_{2}$ depends on $\gamma, \mu \rho$ and $\delta$. However $D_{1} \widetilde{u}=\sqrt{1+|\bar{D} \widetilde{u}|^{2}} / v \geqq 0$, as one easily checks from the relation $\tilde{\nu} Q=\nu$. Thus we can apply (3.9) to $D_{1} \tilde{u}$. Because of (2.6) we then deduce the required Harnack inequality for $v$. This completes the proof of Theorem $1^{\prime 2}$.

An unsatisfactory feature of Theorem 2 is that the hypotheses on $b^{*}$ are such as to exclude certain important examples. For instance, the capillary surface equation

$$
\sum_{i=1}^{2} D_{i}\left(\frac{D_{i} u}{\sqrt{1+.|D u|^{2}}}\right)=\kappa u, \quad \kappa>0
$$

is excluded from the above discussion. This defect is remedied in the following theorem, in which the following condition is assumed in place of (3.3):

$$
\begin{aligned}
-\rho \sqrt{1+|p|^{2}} \frac{\partial b^{*}}{\partial z}(x, z, \mu)+\rho \sum_{k=1}^{2}\left|\frac{\partial}{\partial x_{k}} b^{*}(x, z, \mu)\right| \\
+|\mu-\bar{\mu}|^{-1}\left|b^{*}(x, z, \mu)-b^{*}(x, z, \bar{\mu})\right| \leqq \delta \rho^{-1}
\end{aligned}
$$

for all $(x, z) \in \Omega \times R$ and $\mu, \bar{\mu} \in S_{+}^{2}$ with $\mu \neq \bar{\mu}$ and

$$
\mu=\frac{(-p, 1)}{\sqrt{1+|p|^{2}}}, \quad p \in \boldsymbol{R}^{2} .
$$

THEOREM $2 "$. If $D_{\rho}\left(x_{0}\right) \subset \Omega$, if $u \geqq 0$ on $D_{\rho}\left(x_{0}\right)$ and if (3.2), (3.10) hold, then

$$
\left|D u\left(x_{0}\right)\right| \leqq c_{1} \exp \left\{c_{2} u\left(x_{0}\right) / \rho\right\},
$$

where $c_{1}, c_{2}$ are constants depending on $\gamma, \mu \rho$ and $\delta$.

Proof. In the proof $c_{1}, c_{2}, \cdots$ will denote constants depending only on $\gamma, \mu \rho$ and $\delta$.

We consider the same two cases as in the proof of Theorem 1. In Case I the required result is trivially satisfied. The argument for Case II begins as before, except that in place of (3.7) we now deduce that $\psi=D_{1} \tilde{u}$ is a supersolution of the equation (3.7). That

2 Actually we have only proved $\sup _{s_{\theta \rho}\left(x_{0}\right)} v \leqq c \inf s_{s_{\theta}\left(x_{0}\right)} v$ for some $\theta \in(0,1)$ depending on $\gamma, \mu \rho$ and $\delta$. The required result (with $\theta=1 / 2$ ) follows because we can vary $\mathrm{X}_{0}$. 
is, we deduce

$$
\int_{U}\left(\sum_{i, j=1}^{2} \alpha_{i j} D_{j} \psi D_{i} \zeta+\sum_{i=1}^{2} \beta_{i} D_{i} \psi \zeta+\tau \psi \zeta\right) d \xi \geqq 0
$$

for any nonnegative $\zeta \in C_{0}^{1}(U)$, where (3.8) still holds. Replacing $\zeta$ by $\zeta^{2} / \psi$, we then deduce that

$$
\begin{aligned}
\int_{U}\left\{\zeta^{2} \sum_{j, i=1}^{2} \alpha_{i j} D_{i} w D_{j} w\right. & +2 \zeta \sum_{i, j=1}^{2} \alpha_{i j} D_{i} w D_{j} \zeta \\
& \left.+\zeta^{2}\left(\sum_{i=1}^{2} \beta_{i} D_{i} w-\tau\right)\right\} d \xi \leqq 0
\end{aligned}
$$

for all $\zeta \in C_{0}^{1}(U)$, where $w=\log \psi^{-1}$. Using well-known arguments from the De Giorgi-Nash theory of uniformly elliptic equations, one can easily deduce from (3.12) that

$$
w(0) \leqq c_{1}+c_{2} \int_{D^{+}} w d \xi,
$$

where $D^{+}=\left\{\xi \in D_{\theta \rho / 4}(0): w(\xi)>1\right\}$. The remainder of the proof consists is estimating the integral on the right of (3.13). We begin by noting that inequality (3.11) implies

$$
\int_{D_{\theta \rho / 2}\left(X_{0}\right)}|D w|^{2} d \xi \leqq c_{3} .
$$

If we let $\omega$ be defined by $\omega=\log v$ on $M$, then it is clear (by (2.6)) that this last inequality implies

$$
\int_{S_{\theta \rho 2}\left(X_{0}\right)}|\delta \omega|^{2} d A \leqq c_{4}{ }^{3}
$$

where $\delta$ denotes the tangential gradient operator on $M$. Now define

$$
E=\left\{X=(x, u(x)) \in M \cap\left(D_{\theta \rho / 4}\left(x_{0}\right) \times \boldsymbol{R}\right): u(x)<u\left(x_{0}\right)+\theta \rho / 4\right\} .
$$

We can choose points $X_{1}, \cdots, X_{N} \in E$ such that $E \subset \bigcup_{i=1}^{N} S_{\theta \rho / 4}\left(X_{i}\right)$ and

$$
N \leqq c_{5}\left(1+u\left(x_{0}\right) / \rho\right) \text {. }
$$

Using (3.14) with $X_{i}$ in place of $X_{0}$, summing over $i$, and also using (3.15), we then deduce that

$$
\int_{E}|\delta \omega|^{2} d A \leqq c_{6}\left(1+u\left(x_{0}\right) / \rho\right) .
$$

We now recall the fact (see e.g. [8], (4.6)) that

$$
\left(1-\nu_{3}^{2}\right) \min \left\{\kappa_{1}^{2}, \kappa_{2}^{2}\right\} \leqq\left|\delta \nu_{3}\right|^{2}
$$

${ }^{3}$ Such a equality also holds, of course, if $\left|D u\left(x_{0}\right)\right|<2$; in this case one simply essentially repeats the previous argument without introducing new coordinates $\xi$. 
at each point of $M$. Hence at points where $\nu_{3}^{2}<1 / 2$ (that is, where $\omega>\log \sqrt{2}$ ) we have

$$
\kappa_{1}^{2}+\kappa_{2}^{2} \leqq c_{7}\left(\left|\delta \nu_{3}\right|^{2}+\mu^{2}\right) \leqq c_{7}\left(|\delta \omega|^{2}+\mu^{2}\right)
$$

by virtue of (1.7). Hence, since $\log \sqrt{2}<1$,

$$
\int_{E \cap\{X: \omega(X)>1\}} H^{2} d A \leqq c_{9}\left(\int_{E}|\delta \omega|^{2} d A+\mu^{2}|E|\right), \quad H=\kappa_{1}+\kappa_{2} .
$$

Also, we know that

$$
|E| \leqq c_{10}\left(1+u\left(x_{0}\right) / \rho\right) \rho^{2}
$$

by virtue of (3.15) and the area bounds $\left|S_{\theta \rho / 4}\left(X_{i}\right)\right| \leqq c_{11} \rho^{2}$. Next we have, by the first variation formula for the surface $M$,

$$
\int_{M} \delta_{3} h d A=\int_{M} \nu_{3} H h d A,
$$

whenever $h$ is a $C^{1}$ function with compact support in $M$. In particular, we can choose $h$ of the form

$$
h(X)=f\left(x_{3}\right) g(\omega(X)) \zeta\left(x_{1}, x_{2}\right), \quad X=\left(x_{1}, x_{2}, x_{3}\right) \in M,
$$

where $f, g$ are $C^{1}(R)$ functions and $\zeta \in C^{1}\left(\boldsymbol{R}^{2}\right)$ with

$$
\left\{\begin{array}{l}
g(\omega)=\omega \text { for } \omega>2, g(\omega)=0 \text { for } \omega<1, \text { and } 0 \leqq g^{\prime}(\omega) \leqq L \text { for } \omega \in R \\
f(t) \equiv 0 \text { for } t>u\left(x_{0}\right)+\sigma, f^{\prime}(t) \equiv-1 \text { for } t \in\left(u\left(x_{0}\right)-\sigma / 2, u\left(x_{0}\right)+\sigma / 2\right), \\
0 \leqq-f^{\prime}(t) \leqq 2 \text { for } t \in R, f(t) \equiv \sigma \text { for } t<u\left(x_{0}\right)-\sigma \\
\zeta(x) \equiv 1 \text { for } x \in D_{\sigma / 2}\left(x_{0}\right), \zeta(x) \equiv 0 \text { for } x \in \sim D_{\sigma}\left(x_{0}\right), \\
|D \zeta(x)| \leqq 3 / \sigma \text { for } x \in R^{2} . \text { Here } \sigma=\theta \rho / 4 .
\end{array}\right.
$$

With such a choice of $h$ one easily deduces

$$
\int_{S_{\theta_{\rho} / 8\left(X_{0}\right)}} \omega d A \leqq c_{12}\left\{\rho \int_{E \cap\{X: \omega(X)>1\}}\left(|\delta \omega|+\nu_{3} \omega|H|\right) d A+|E|\right\} .
$$

Since $\nu_{3} \omega<1$ we then use (3.16), (3.18) and (3.19) to deduce

$$
\int_{S_{\theta_{\rho} / 8}\left(X_{0}\right)} \omega d^{d} A \leqq c_{13}\left(1+u\left(x_{0}\right) / \rho\right) \text {. }
$$

The required result now follows from this and (3.13). (One needs to note that $S_{\theta \rho / 8}\left(X_{0}\right) \supset\left\{(\xi, \tilde{u}(\xi)) Q: \xi \in D_{\theta \rho / 16}(0)\right\}$ by virtue of (2.6).)

Next we present an analogue of Theorem 8. Note that the estimate obtained in the theorem here is weaker than that of Theorem 8 in that there is no factor of $\left(v\left(X_{0}\right)\right)^{-2}$ on the right hand side. (Consideration of graphs with constant mean curvature shows that one cannot expect to have such a factor on the right hand side in the 
nonhomogeneous case.)

THEOREM 8'. Suppose $D_{\rho}\left(x_{0}\right) \subset \Omega$, suppose (2.3) holds, and suppose

$$
\left|b^{*}(X, \mu)-b^{*}(\bar{X}, \bar{\mu})\right| \leqq \rho^{-1} \delta\{|X-\bar{X}| / \rho+|\mu-\bar{\mu}|\}^{\alpha}
$$

for all $\dot{X}, \bar{X} \in S_{\rho}\left(X_{0}\right)$ and all $\mu, \bar{\mu} \in S_{+}^{2}$, where $\delta>0$ and $\alpha \in(0,1)$ are constants. Then

$$
\left(\kappa_{1}^{2}+\kappa_{2}^{2}\right)\left(X_{0}\right) \leqq c \rho^{-2},
$$

where $c$ is a constant depending only on $\gamma, \mu \rho, \delta$ and $\alpha$.

Proof. As in the proof of Theorem 8, we introduce new coordinates as in (2.4) and infer from (1.1) an equation like (2.8) for the function $\tilde{u}$ of (2.5), (2.6). However notice that here we have to use the inequality of Theorem 3 ' instead of the stronger inequality of Theorem 3. Also, the equation for $\widetilde{u}$ corresponding to (2.8) now has the form

$$
\sum \widetilde{a}_{i j}(\xi) D_{i j} \widetilde{i}=\widetilde{b}(\xi) \text { on } U,
$$

where (2.9) still holds, where $|\tilde{b}| \leqq \mu$ on $U$ and where (by virtue of (2.3), (3.22) and the estimate of Theorem $3^{\prime}$ )

$$
\sum_{i, j=1}^{2}\left|\widetilde{a}_{i j}(\xi)-\widetilde{a}_{i j}(\bar{\xi})\right|+\rho|\widetilde{b}(\xi)-\widetilde{b}(\bar{\xi})| \leqq c\{|\xi-\bar{\xi}| / \rho\}^{\tau}
$$

for $\xi, \bar{\xi} \in D_{\theta \rho}(0)$. Here $\theta \in(0,1), \tau \in(0,1)$ and $c>0$ depend on $\alpha, \delta, \gamma$ and $\mu \rho$. Then by applying Schauder's interior estimate as in the proof of Theorem 8, we obtain the required inequality.

The next theorem generalizes Theorem 6; notice that there are no continuity hypotheses on the functions $a_{i j}, b$ in the theorem.

Theorem 6'. Suppose $\Omega=\boldsymbol{R}^{2} \sim K$, where $K$ is compact, and suppose that

$$
\left|b^{*}(X, \mu)\right| \leqq \mu_{0} /|X|^{1+\tau}, \quad X \in \boldsymbol{R}^{3} \sim\{0\}, \mu \in S_{+}^{2},
$$

where $\mu_{0}>0$ and $\tau \in(0,1)$ are given constants. Then there is a vector $\nu^{0}=\left(\nu_{1}^{0}, \nu_{2}^{0}, \nu_{3}^{0}\right) \in \bar{S}_{+}^{2}$ such that

$$
\nu(X)\left(=\left(1+|D u(x)|^{2}\right)^{-1 / 2}(-D u(x), 1)\right) \longrightarrow \nu^{0}
$$

uniformly for $|x| \rightarrow \infty$. In case $b \equiv 0$, then $\nu_{3}^{0}>0$ and hence there is an $a \in \boldsymbol{R}^{2}$ such that $D u(x) \rightarrow a$ uniformly for $|x| \rightarrow \infty$.

Proof. The proof relies havily on the techniques of [8]. In the 
proof, constants which depend at most on $\gamma, \rho_{0}, \mu_{0}$ and $\tau$ will be denoted $c_{1}, c_{2}, \cdots$.

To begin, let $R_{0}$ be such that $K \subset D_{R_{0}}(0)$, define

$$
\rho_{0}=\sup \left\{\left(u^{2}(x)+|x|^{2}\right)^{1 / 2}: x \in \partial D_{R_{0}}(0)\right\}
$$

and for $R>\rho>\rho_{0}$ let $T_{\rho, R}$ be defined by

$$
T_{\rho, R}=\{X \in M: \rho<|X|<R\} .
$$

We will repeatedly make use of the fact that, for $\rho \geqq \sigma>0, T_{\rho, \sigma+\sigma}$ is covered by $S_{\sigma}\left(X_{1}\right), \cdots, S_{\sigma}\left(X_{N}\right)$, where $X_{1}, \cdots, X_{N}$ are points of $T_{\rho, \sigma+\sigma}$ and $N \leqq c \rho / \sigma$, where $c$ is an absolute constant.

From the discussion of $\S 4$ of [8], have the identity

$$
\kappa_{1} \kappa_{2} d A=d \omega^{*}
$$

where $\kappa_{1}, \kappa_{2}$ are the principal curvatures of $M$ and $\omega^{*}$ is the 1 -form on $M$ defined by

$$
\omega^{*}=\left(1+\nu_{3}\right)^{-1}\left(-\nu_{2} d \nu_{1}+\nu_{1} d \nu_{2}\right) .
$$

Using Stokes' Theorem we then have, for almost all $\rho, R$ with $\rho_{0}<$ $\rho<R$,

$$
\int_{T_{\rho, R}} \zeta^{2} \kappa_{1} \kappa_{2} d A=-\int_{T_{\rho, R}} d \zeta^{2} \wedge \omega^{*}+\int_{\partial T_{\rho, R}} \zeta^{2} \omega^{*} .
$$

Using (1.7) (and noting that (3.23) implies that we can take $\mu$ to be the variable quantity $\left.\mu_{0} /|X|^{1+\tau}\right)$, we then deduce

$$
\begin{aligned}
\left|\Lambda_{1}\right|^{-1} \int_{T_{\rho, R}} \zeta^{2}|\delta \nu|^{2} d A \leqq & \left|\int_{T_{\rho, R}} 2 \zeta d \zeta \wedge \omega^{*}\right|+\left|\int_{\partial T_{\rho \cdot R}} \zeta^{2} \omega^{*}\right| \\
& +\Lambda_{2}\left|\Lambda_{1}\right|^{-1} \int_{T_{\rho, R}} \zeta^{2}|X|^{-2-2 \tau} d A .
\end{aligned}
$$

If $\zeta$ is chosen so that $\zeta \equiv 0$ on $\partial T_{\rho, R}$, then this gives

$$
\int_{T_{\rho, R}} \zeta^{2}|\delta \nu|^{2} d A \leqq c_{1} \int_{T_{\rho, R}}\left(\zeta|\delta \nu||\delta \zeta|+\zeta^{2}|X|^{-2-2 \tau}\right) d A,
$$

which clearly implies (by Cauchy's inequality)

$$
\int_{T_{\rho, R}} \zeta^{2}|\delta \nu|^{2} d A \leqq c_{2} \int_{T_{\rho, R}}\left(|\delta \zeta|^{2}+\zeta^{2}|X|^{-2-2 \tau}\right) d A
$$

Next we note that because of the area bounds

$$
\left|S_{o}\left(X_{0}\right)\right| \leqq c_{3} \sigma^{2},
$$

which by Lemma (3.1) of [8] are valid when $X_{0} \in M$ and $\left|X_{0}\right|>\rho_{0}+$ 
$2 \sigma$, we can deduce

$$
\left|T_{\rho, \rho+\sigma}\right| \leqq c_{4} \rho \sigma, \quad \rho>2 \rho_{0}, 0<\sigma \leqq \rho .
$$

By using a suitable choice of $\zeta$ together with (3.28), it is now not difficult to see that (3.26) implies

$$
\int_{T_{2 \rho, R / 2}}|\delta \nu|^{2} d A \leqq c_{5}, \quad R>4 \rho .
$$

Thus (since $c_{5}$ is independent of $R$ ) we deduce

$$
\int_{T_{\rho, \infty}}|\delta \nu|^{2} d A \leqq c_{5}
$$

for $\rho>2 \rho_{0}$. By again choosing $\zeta$ appropriately, we can now deduce from (3.25) and (3.28)

$$
\int_{T_{\rho, \infty}}|\delta \nu|^{2} d A \leqq c_{6}\left\{\left|\int_{\partial T_{\rho, \infty}} \omega^{*}\right|+\rho^{-2 \tau}\right\}
$$

By a straightforward modification of the argument of [8], Theorem (2.1), we can infer from this that

$$
\hat{\mathscr{D}}(\rho) \leqq-c_{7} \rho \hat{\mathscr{D}}^{\prime}(\rho)+c_{8} \rho^{-2 \tau},
$$

for almost all $\rho>4 \rho_{0}$, where

$$
\hat{\mathscr{D}}(\rho)=\int_{T_{\rho, \infty}}|\delta \nu|^{2} d A .
$$

(It is necessary to note the fact that

$$
-\left.\frac{d}{d \rho} \int_{T_{\rho, \infty}}|\delta| X\right|^{2} d A \leqq c_{9}\left\{\rho^{-1}\left|T_{\rho, 2 \rho}\right|+\int_{T_{\rho, 2 \rho}}|H| d A\right\},
$$

where $H=\kappa_{1}+\kappa_{2}$. This follows by using the first variation formula for $M$ in a manner similar to the argument leading to (A.2) to (A.2) of [8].) By integrating (3.32) from $4 \rho_{0}$ to $\rho$, we now conclude

$$
\hat{\mathscr{D}}(\rho) \leqq c_{10} \rho^{-\beta}, \quad \rho>4 \rho_{0},
$$

where $\beta \in(0,2 \tau)$ depends only on $\gamma, \rho_{0}, \mu_{0}$ and $\tau$. In particular we note that

$$
\int_{S_{\rho}\left(X_{0}\right)}|\delta \nu|^{2} d A \equiv \mathscr{D}\left(X_{0}, \rho\right) \leqq c_{10} \rho^{-\beta}
$$

for all $X_{0} \in M$ with $\left|X_{0}\right|>2 \rho>8 \rho_{0}$.

Now an examination of the proof of the Hölder estimate of theorem (in particular see (2.11), (2.12) of [8]) will show that in the present setting we can assert 


$$
\left|\nu(X)-\nu\left(X_{0}\right)\right| \leqq c_{11}\left\{\mathscr{D}\left(\rho, X_{0}\right)+\rho^{-2 \tau}\right\}^{1 / 2}\{\sigma / \rho\}^{\alpha}
$$

for any $X \in S_{\sigma}\left(X_{0}\right), \sigma \in(0, \rho)$. Combining (3.33)' and (3.34), with $\sigma=\rho$, gives

$$
\left|\nu(X)-\nu\left(X_{0}\right)\right| \leqq c_{12} \rho^{-\beta^{\prime}}, \quad X \in S_{\rho}\left(X_{0}\right), \beta^{\prime}=\beta / 2,
$$

and this clearly implies (since we can vary $X_{0}$ )

$$
\sup _{X, \bar{X} \in T \rho, 2 \rho}|\nu(X)-\nu(\bar{X})| \leqq c_{13} \rho^{-\beta^{\prime}}
$$

for any $\rho>4 \rho_{0}$. Then for any integer $k \geqq 1$ we have

$$
\sup _{X, \bar{X} \in T_{\rho, 2^{k}}}|\nu(X)-\nu(\bar{X})| \leqq c_{13} \rho^{-\beta^{\prime}}\left(\sum_{r=0}^{k-1} 2^{-r \beta^{\prime}}\right),
$$

where upon we deduce that

$$
\sup _{x, \bar{X} \in T_{\rho}, \infty}|\nu(X)-\nu(\bar{X})| \leqq c_{14} \rho^{-\beta^{\prime}} .
$$

The first conclusion of the theorem clearly follows from this.

We now consider the case $b \equiv 0$. We then have (1.7) with $\Lambda_{2}=0$, so that we can use the theory developed in $\S 4$ of [8]. In particular we use the identity

$$
\left(\left(1-\nu_{3}\right) \gamma\left(\nu_{3}\right)\right)^{\prime} \kappa_{1} \kappa_{2} d A=-d\left(\gamma\left(\nu_{3}\right)\left(1+\nu_{3}\right)^{-1}\left(-\nu_{2} d \nu_{1}+\nu_{1} d \nu_{2}\right)\right),
$$

where $\gamma$ is an arbitrary $C^{1}(R)$ function. (This identity is the pointwise version of identity (4.5) of [8], as one easily checks by using Stokes' Theorem.) We multiply each side of (3.35) by a cut-off function $\zeta^{2}$ which vanishes for $|X|>R$, and integrate (3.35) over $T_{\rho, \infty}$. After using Stokes' Theorem, this gives

$$
\begin{aligned}
\int_{T_{\rho}, \infty} \zeta^{2}\left(\left(1-\nu_{3}\right) \gamma\left(\nu_{3}\right)\right)^{\prime} \kappa_{1} \kappa_{2} d A \\
=-\int_{\partial T_{\rho}, \infty} \zeta^{2} \gamma\left(\nu_{3}\right)\left(1+\nu_{3}\right)^{-1}\left(-\nu_{2} d \nu_{1}+\nu_{1} d \nu_{2}\right) \\
\quad-\int_{T_{\rho}, \infty} 2 \zeta \gamma\left(\nu_{3}\right)\left(1+\nu_{3}\right)^{-1} d \zeta \wedge\left(-\nu_{2} d \nu_{1}+\nu_{1} d \nu_{2}\right) .
\end{aligned}
$$

Now from Theorem 1 we know that

$$
\sup _{\partial T_{p}, \infty} \nu_{3} \leqq c_{15} \inf _{\partial T_{\rho}, \infty} \nu_{3}
$$

for each $\rho>2 \rho_{0}$.

Also if there exists a sequence $\left\{X_{j}\right\} \subset M$ with $\left|X_{j}\right| \rightarrow \infty$ and with $\left\{\nu_{3}^{-1}\left(X_{j}\right)\right\}$ bounded, we could immediately deduce $\nu_{3}^{\urcorner}>0$, and the required Eresult would be established. We may therefore assume $\nu_{3}(X)<1 / 2$ 
for all $|X|>\rho_{1}$, where $\rho_{1}>\rho_{0}$ is a fixed constant. Then for $\rho>\rho_{1}$ we can replace $\gamma\left(\nu_{3}\right)$ in $(3.36)$ by $\left(\nu_{3}\right)^{-1} /\left(1-\nu_{3}\right)$, thus giving

$$
\begin{aligned}
\int_{T_{\rho, \infty}} \zeta^{2} \nu_{3}^{-2} \kappa_{1} \kappa_{2} d A= & -\int_{T_{\rho, \infty}} \nu_{3}^{-1}\left(1-\nu_{3}^{2}\right)^{-1} d \zeta^{2} \wedge\left(-\nu_{2} d \nu_{1}+\nu_{1} d \nu_{2}\right) \\
& -\int_{\partial T_{\rho, \infty}} \zeta^{2} \nu_{3}^{-1}\left(1-\nu_{3}^{2}\right)^{-1}\left(-\nu_{2} d \nu_{1}+\nu_{1} d \nu_{2}\right)
\end{aligned}
$$

Using inequality (4.8) of [8] and also using Cauchy's inequality, one easily checks that (3.38) implies

$$
\int_{T_{\rho, \infty}} \zeta^{2}|\delta w|^{2} d A \leqq c_{16}\left\{\int_{T_{\rho}, \infty}|\delta \zeta|^{2} d A+\int_{\partial T_{\rho}}|\delta w| \zeta^{2} d s\right\} .
$$

Here, and subsequently, $w=\log \nu_{3}^{-1}$. Choosing $\zeta(X) \equiv 1$ for $|X|<$ $R / 2$ and $\zeta(X) \equiv 0$ for $|X|>R$, and letting $R \rightarrow \infty$, we then deduce that

$$
\int_{T_{\rho, \infty}}|\delta w|^{2}<\infty
$$

for $\rho>\rho_{1}$. Making a similar choice of $\zeta$ in (3.38) and again letting $R \rightarrow \infty$, we can conclude, for almost all $\rho>\rho_{1}$,

$$
\int_{T_{\rho, \infty}}|\delta w|^{2} d A \leqq c_{17}\left|\int_{\partial T_{\rho, \infty}}-\omega_{1} d \nu_{1}+\omega_{2} d \nu_{2}\right|
$$

where

$$
\omega_{i}=\nu_{i} \nu_{3}^{-1}\left(1-\nu_{3}^{2}\right)^{-1}, \quad i=1,2 .
$$

Now for almost all $\rho>\rho_{1}$ we have $\partial T_{\rho, \infty}=\bigcup_{i=1}^{N(\rho)} \Gamma_{\rho}^{(j)}$, where $N(\rho)$ is a positive integer and $\Gamma_{\rho}^{(j)}$ is a smooth Jordan curve such that $\delta|X|$ does not vanish of $\Gamma_{\rho}^{(j)}$. Since $\int_{\Gamma_{\rho}^{(j)}} d \nu_{i}=0$, we can write $(3.40)$ in the form

$$
\begin{aligned}
\int_{T_{\rho, \infty}}|\delta w|^{2} d A \leqq & c_{17} \mid \sum_{j=1}^{N(\rho)} \int_{\Gamma_{\rho}^{(j)}}-\left(\omega_{1}-\omega_{1}\left(X^{(j)}\right)\right) d \nu_{1} \\
& +\left(\omega_{2}-\omega_{2}\left(X^{(j)}\right)\right) d \nu_{2} \mid
\end{aligned}
$$

where $X^{(j)}$ denotes a fixed point on $\Gamma_{\rho}^{(j)}$. Now

$$
\sup _{\Gamma_{\rho}^{(j)}}\left|\omega_{i}-\omega_{i}\left(X^{(j)}\right)\right| \leqq \int_{\Gamma_{\rho}^{(j)}}\left|\frac{d \omega_{i}}{d s}\right| d s \leqq c_{18} \int_{\Gamma_{\rho}^{(j)}} \nu_{3}^{-2}|\delta \nu| d s
$$

Combining (3.41) and (3.42), we obtain

$$
\int_{T_{\rho, \infty}}|\delta w|^{2} d A \leqq c_{19}\left(\int_{\partial T_{\rho, \infty}}|\delta \nu| d s\right)^{2} \sup _{\partial T_{\rho} \infty} \nu_{3}^{-2} .
$$


In view of inequality (4.8) of [8] and inequality (3.37) above, we then deduce

$$
\int_{T_{\rho, \infty}}|\delta w|^{2} d A \leqq c_{20}\left(\int_{\partial T_{\rho, \infty}}|\delta w| d s\right)^{2} .
$$

Using (3.32) and the Hölder inequality one can then use an argument like that of [8] Theorem (2.1) (the argument is like that needed to obtain the estimate (3.33) above); we thus obtain

$$
\int_{T_{\rho, \infty}}|\delta w|^{2} d A \leqq c_{21} \rho^{-\beta^{\prime}}
$$

where $\beta^{\prime} \in(0,1)$ depends only on $\rho_{0}$ and $\gamma$. In particular we have

$$
\int_{S_{\rho}\left(X_{0}\right)}|\delta w|^{2} d A \leqq c_{21} \rho^{-\beta^{\prime}}
$$

whenever $Y_{0} \in M$ and $\left|X_{0}\right|>2 \rho, \rho>\rho_{1}$. Using this last inequality in combination with the inequalities (4.15), (4.16) of [8], we then deduce

$$
\sup _{s_{\rho, 2}\left(X_{0}\right)} w-\inf _{s_{\rho, 2}\left(X_{0}\right)} w \leqq c_{22} \rho^{-\beta^{\prime} / 2} .
$$

In view of the arbitrariness of $X_{0}$, this gives

$$
\sup _{T_{\rho, 2 \rho}} w-\inf _{T_{\rho, 2 \rho}} w \leqq c_{23} \rho^{-\beta^{\prime} / 2} .
$$

Iterating, we obtain

$$
\sup _{T_{\rho, 2^{k} \rho}} w-\inf _{T_{\rho, 2^{k} \rho}} w \leqq c_{23} \rho^{-\beta^{\prime} / 2}\left(\sum_{r=0}^{\infty} 2^{-r \beta^{\prime} / 2}\right)
$$

for each integer $k \geqq 1$. Hence

$$
\sup _{T_{\rho}, \infty} w-\inf _{T_{\rho}, \infty} w \leqq c_{24} \rho^{-\beta^{\prime}} ;
$$

that is, $w$ is bounded for $|X|>\rho_{1}$. The theorem now follows.

It is not clear whether or not an estimate like that obtained in Theorem 5 holds for general equation (1.1)-(1.3), (3.2), (3.3), (3.23). However, for a class of divergence-form equations (with $n \geqq 2$ independent variables) such a theorem is obtained in [10]; from the discussion of $\S 1$ of [10] it is clear that the structural conditions imposed there certainly hold in case the equation arises as the nonparametric Euler-Lagrange equation (equation (A.6) in Appendix 1) of an elliptic parametric functional with integrand $F(X, q)$ in case $F(X, q)$ is independent of $X$. More generally, it suffices that $F(X, q)=$ $F(x, z, q)$ is such that $F_{z}(x, z, q) \equiv 0$. (Actually the condition (1.3) of [10] is not quite stated in a weak enough form to include this latter case; however, one can check that all the results of [10] 
remain valid if the first inequality of [10], (1.3) is replaced by the weaker condition

$$
\left|\sum_{i=1}^{n} u_{x_{i}} D_{i k}\right| \leqq \beta_{5}, \quad \sum_{i, k=1}^{n}\left|D_{i k}\right| \leqq \beta_{5},
$$

where (in the notation of [10])

$$
D_{i k}=u_{x_{l}} A_{i x_{l} p_{k}}+u_{x_{k}} A_{i z}+u_{x_{i}} B_{p_{k}}+|D u|^{2} A_{i z} p_{k},
$$

and this condition is weak enough to include the case when $F$ in (A.6) satisfies $F_{z}(x, z, q) \equiv 0$.)

Finally we state the following analogue of Theorem 6; a proof will be found in [9].

THEOREM. Suppose $D_{\rho}\left(x_{0}\right) \sim\left\{x_{0}\right\} \subset \Omega$. Then the closure $\bar{M}$ (taken in $\Omega \times \boldsymbol{R})$ of the graph of $u$ is a $C^{1, \alpha}$ surface such that $\left(\bar{D}_{o}\left(x_{0}\right) \times \boldsymbol{R}\right) \cap \bar{M}$ is compact for each $\sigma<\rho$.

One can show by example that even though the graph of $u$ can thus be extended to a $C^{1, \alpha}$ surface in $\boldsymbol{R}^{3}$, nevertheles the function $u(x)$ (as a function of $x \in \Omega$ ) may have no $C^{1}$ extension to $D_{\rho}\left(x_{0}\right)$. (Because it may happen that $|D u(x)|$ is unbounded for $x$ in a neighbourhood of $x_{0}$.)

\section{APPENDIX: Elliptic Parametric Functionals.}

Let $\Omega$ be a bounded domain in $R^{2}$ and consider the functional $I$, defined for $C^{1}$ mappings $Y=\left(Y_{1}, Y_{2}, Y_{3}\right): \bar{\Omega} \rightarrow R^{3}$ by

$$
I(Y)=\int_{\Omega} G\left(x, Y, D_{1} Y, D_{2} Y\right) d x,
$$

where $G=G(x, X, p)$ is a given continuous function of $(x, X, p) \in$ $\boldsymbol{R}^{2} \times \boldsymbol{R}^{3} \times \boldsymbol{R}^{6}$. (Here of course $D_{i} Y=\left(D_{i} Y_{1}, D_{i} Y_{2}, D_{i} Y_{3}\right)$ for $i=1,2$.) Now let us consider the possibility that $I$ remains invariant under orientation preserving diffeomorphism of $\boldsymbol{R}^{2}$; that is, whenever $\psi$ is a diffeomorphism of $\boldsymbol{R}^{2}$ onto itself with positive Jacobian, we would have

$$
\int_{\Omega} G\left(\xi, \widetilde{Y}(\xi), D_{1} \widetilde{Y}(\xi), D_{2} \widetilde{Y}(\xi)\right) d \xi=\int_{\Omega} G\left(x, Y(x), D_{1} Y(x), D_{2} Y(x) d x,\right.
$$

where $\Omega^{\prime}=\psi(\Omega)$ and $\tilde{Y}=Y \circ \psi^{-1}$. A simple computation (cf. [7], p. 349) shows that this would be true for all such diffeomorphisms $\psi$ and domains $\Omega$ if and only if there is a real-valued function $F$ on $\boldsymbol{R}^{3} \times \boldsymbol{R}^{3}$ such that 
(A2)

$$
G(x, X, p)=F(X, P), \quad(x, X, p) \in R^{2} \times R^{3} \times R^{6},
$$

where

$$
P=\left(p_{3} p_{5}-p_{2} p_{6}, p_{1} p_{6}-p_{3} p_{4}, p_{2} p_{4}-p_{1} p_{5}\right) ;
$$

and

$$
F(X, \lambda q)=\lambda F(X, q), \quad(X, q) \in \boldsymbol{R}^{3} \times \boldsymbol{R}^{3}, \quad \lambda>0 .
$$

Note in particular that (A2) implies that $G(x, X, p)$ cannot depend on $x$; that is, $G(x, X, p)=G(0, X, p)$ for $(x, X, p) \in \boldsymbol{R}^{2} \times \boldsymbol{R}^{3} \times \boldsymbol{R}^{6}$. In case $p=\left(D_{1} Y, D_{2} Y\right)$, where $Y$ is a $C^{1}$ map from $\bar{\Omega}$ into $R^{3}, P$ is given by

$$
\begin{array}{r}
P=\left(D_{1} Y_{3} \cdot D_{2} Y_{2}-D_{1} Y_{2} \cdot D_{2} Y_{3}, D_{1} Y_{1} \cdot D_{2} Y_{3}-D_{1} Y_{3} \cdot D_{2} Y_{1},\right. \\
\left.D_{1} Y_{2} \cdot D_{2} Y_{1}-D_{1} Y_{1} \cdot D_{2} Y_{2}\right) .
\end{array}
$$

As is well known, in case $Y$ is one-to-one and such that the Jacobian matrix $\left[D_{j} Y_{i}(x)\right]$ has rank 2 for each $x \in \Omega$, this last identity can be written

$$
P=\chi \nu,
$$

where $\nu$ is the unit normal of the embedded surface $S=\{Y(x) \mid x \in \Omega\}$ and $\chi$ is the area magnification factor of the mapping $Y$. Thus, assuming that we orient $S$ with unit normal $\nu$ such that $\chi>0$, we can write

$$
I(Y)=\int_{S} F(X, \nu(X)) d A(X)
$$

that is, we can express $I(Y)$ completely in terms of the oriented surface $S$ and independently of the particular mapping $Y$ that is used to represent $S$. Through this discussion we are led to consider the functional $J$, defined for any smooth oriented surface $S$ in $R^{3}$ having finite area, by

$$
J(S)=\int_{S} F(X, \nu(X)) d A(X) ;
$$

this functional has the property that $J(S)=I(Y)$ whenever $Y$ is a one-to-one $C^{1}$ mapping from $\Omega$ into $\boldsymbol{R}^{3}$ such that $\left[D_{j} Y_{i}\right.$ ] has rank 2 at each point of $\Omega$ and $S=\{Y(x) \mid x \in \Omega\}$.

If $F$ satisfies (A3), we call a functional of the form (A4) a parametric functional. The functional $J$ is called elliptic if $F$ is $C^{2}$ on $\boldsymbol{R}^{3} \times\left(\boldsymbol{R}^{3}-\{0\}\right)$ and if the convexity condition

$$
|q| D_{q_{i} q_{j}} F(X, q) \xi_{i} \xi_{j} \geqq\left|\xi^{\prime}\right|^{2}, \quad \xi^{\prime}=\dot{\xi}-\left(\xi \cdot \frac{q}{|q|}\right) \frac{q}{|q|},
$$


holds for all $X \in \boldsymbol{R}^{3}, q \in \boldsymbol{R}^{3}-(0)$ and $\xi \in \boldsymbol{R}^{3}$. Notice that, up to a scalar factor, (A5) is the strongest convexity condition possible for $F$ in view of the homogeneity condition (A3).

If we now consider a nonparametric surface $M$ given by

$$
M=\left\{(x, u(x)) \in \boldsymbol{R}^{3} \mid x \in \Omega\right\},
$$

where $u \in C^{2}(\bar{\Omega})$, then, taking $\nu$ to be the downward unit normal $(D u,-1) / \sqrt{1+|D u|^{2}}$, we have

$$
J(S)=\int_{\Omega} F(x, u(x), D u(x),-1) d x .
$$

Notice that here we have used the relation $d A=\sqrt{1+|D u|^{2}} d x$. The expression on the right can be considered as a nonparametric functional, defined for any $u \in C^{2}(\bar{\Omega})$. The Euler-Lagrange equation for this nonparametric functional is

$$
\sum_{i=1}^{2} D_{i}\left[F_{q_{i}}(x, u, D u,-1)\right]-D_{X_{3}} F(x, u, D u,-1)=0 .
$$

By using the chain rule and the homogeneity condition (A3), one can easily check that this equation can be written in the form

$$
a_{i j}(x, u, D u) D_{i j} u=b(x, u, D u),
$$

where

$$
\begin{gathered}
a_{i j}(x, u, D u)=|q| D_{q_{i} q_{j}} F(x, u, D u,-1), \quad i, j=1,2, \\
b(x, u, D u)=-|q| \sum_{i=1}^{3} D_{q_{i} X_{i}}(x, u, D u,-1) .
\end{gathered}
$$

By using (A3), (A5) it is not difficult to check that (1.2), (1.3) hold with constants $\gamma$ and $\mu$ depending on $F$. That is, the nonparametric Euler-Lagrange equation for an elliptic parametric functional is an equation of mean curvature type.

Jenkins [5] and Jenkins-Serrin [6] consider equations of the form (A6) in case $F(X, q)$ does not depend on $X$. Note (A6) has the form (1.1) with $b \equiv 0$ in this case; hence all the results of $\S 2$ apply.

Finally we wish to point out that the functions $a_{i j}^{*}, b^{*}$ introduced in $\S 2$ have a natural interpretation in the present context. In fact one can easily check that in case $a_{i j}, b$ are as in (A7), (A8), then $a_{i j}^{*}, b^{*}$ are given by

$$
\begin{aligned}
a_{i j}^{*}(X, \nu) & =D_{q_{i} q_{j}} F(X, \nu), \quad i, j=1,2,3, \\
b^{*}(X, \nu) & =-|q| \sum_{i=1}^{3} D_{q_{i} X_{i}} F(X, \nu) ;
\end{aligned}
$$


furthermore the conditions (2.3), (3.22) hold automatically with $\delta, \alpha$ determined by $F$, provided that $F \in C^{3}\left(\boldsymbol{R}^{3} \times\left(\boldsymbol{R}^{3}-\{0\}\right)\right)$.

\section{REFERENCES}

1. L. Bers, Isolated singularities of minimal surfaces, Ann. of Math., (2) 53 (1951), 364-386.

2. R. Finn, Equations of minimal surface type, Annals of Math., 60 (1954), 397-416.

3. - Isolated singularities of solutions of non-linear partial differential equations, Trans. Amer. Math. Soc., 75 (1953), 383-404.

4. E. Heinz, Über die Lösungen der Minimal flächengeleichung, Nachr. Akad. Wiss. Göttingen Math. Phy. K1, II (1952), 51-56.

5. H. Jenkins, On 2-dimensional variational problems in parametric form, Arch. Rat. Mech. Anal., 8 (1961), 181-206.

6. H. Jenkins, J. Serrin, Variational problems of minimal surface type I, Arch. Rat. Mech. Anal., 12 (1963), 185-212.

7. C. B. Morrey, Multiple integrals in the calculus of variations, Springer-Verlag.

8. L. Simon, A Hölder estimate for quasiconformal maps between surfaces in Euclidean space. To appear in Acta Math.

9. - Isolated singularities of graphs with quasiconformal Gauss map. In preparation.

10. Global estimates of Hölder continuity for a class of divergence-form elliptic equations, Arch. Rat. Mech. Anal., 56 (1974), 253-272.

11. J. Spruck, Gauss curvature estimates for surfaces of constant mean curvature, Comm. Pure Appl. Math., 27 (1974).

Received August 16, 1976. This research was supported in part by NSF Grant MPS-75-23332 and a Sloan Foundation Fellowship.

STANFORD UNIVERSITY

STANFORD, CA 94305 


\section{PACIFIC JOURNAL OF MATHEMATICS}

EDITORS

RICHARD ARENS (Managing Editor)

University of California

Los Angeles, California 90024

R. A. Beaumont

University of Washington

Seattle, Washington 98105
J. DUGUNDJI

Department of Mathematics

University of Southern Californı

Los Angeles, California 90007

D. Gilbarg and J. Milgram

Stanford University

Stanford, California 94305

\section{ASSOCIATE EDITORS}

E. F. BECKENBACH

B. H. NeumanN

F. WOLF

K. YoSHIDA

\section{SUPPORTING INSTITUTIONS}

UNIVERSITY OF BRITISH COLUMBIA

CALIFORNIA INSTITUTE OF TECHNOLOGY

UNIVERSITY OF CALIFORNIA

MONTANA STATE UNIVERSITY

UNIVERSITY OF NEVADA

NEW MEXICO STATE UNIVERSITY

OREGON STATE UNIVERSITY

UNIVERSITY OF OREGON

OSAKA UNIVERSITY
UNIVERSITY OF SOUTHERN CALIFORNIA

STANFORD UNIVERSITY

UNIVERSITY OF TOKYO

UNIVERSITY OF UTAH

WASHINGTON STATE UNIVERSITY

UNIVERSITY OF WASHINGTON

AMERICAN MATHEMATICAL SOCIETY

NAVAL WEAPONS CENTER 


\section{Pacific Journal of Mathematics}

Vol. 69 , No. 1

May, 1977

V. V. Anh and P. D. Tuan, On starlikeness and convexity of certain analytic

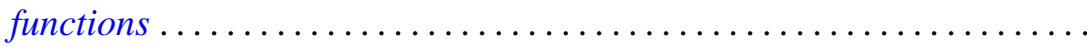

Willard Ellis Baxter and L. A. Casciotti, Rings with involution and the prime

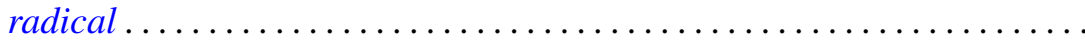

Manuel Phillip Berriozabal, Hon-Fei Lai and Dix Hayes Pettey,

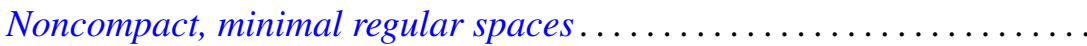

Sun Man Chang, Measures with continuous image law ................

John Benjamin Friedlander, Certain hypotheses concerning

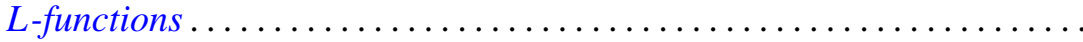

Moshe Goldberg and Ernst Gabor Straus, On characterizations and

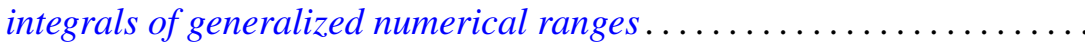

Pierre A. Grillet, On subdirectly irreducible commutative semigroups ...... 55

Robert E. Hartwig and Jiang Luh, On finite regular rings ..............

Roger Hugh Hunter, Fred Richman and Elbert A. Walker, Finite direct sums of cyclic valuated p-groups ........................... 97

Atsushi Inoue, On a class of unbounded operator algebras. III ......... 105

Wells Johnson and Kevin J. Mitchell, Symmetries for sums of the Legendre

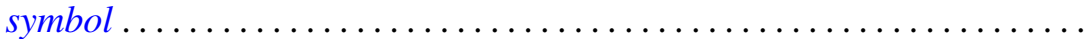

Jimmie Don Lawson, John Robie Liukkonen and Michael William Mislove,

Measure algebras of semilattices with finite breadth

Glenn Richard Luecke, A note on spectral continuity and on spectral properties of essentially $G_{1}$ operators ...............

Takahiko Nakazi, Invariant subspaces of weak-* Dirichlet algebras . .

James William Pendergrass, Calculations of the Schur group ...

Carl Pomerance, On composite $n$ for which $\varphi(n) \mid n-1$. II. . .

Marc Aristide Rieffel and Alfons Van Daele, A bounded operator approach to Tomita-Takesaki theory........................

Daniel Byron Shapiro, Spaces of similarities. IV. $(s, t)$-families ...

Leon M. Simon, Equations of mean curvature type in 2 independent variables.

Joseph Nicholas Simone, Metric components of continuous images of ordered compacta ............................

William Charles Waterhouse, Pairs of symmetric bilinear forms in

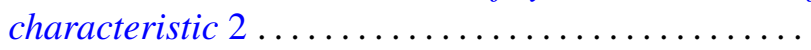

